\title{
Potential Unwinding of Double-stranded DNA Upon Binding to Carbon Nitride
}

\section{Polyaniline $\left(\mathrm{C}_{3} \mathrm{~N}\right)$ Nanosheet}

\author{
Liang Zhao, Zonglin Gu*
}

College of Physical Science and Technology, Yangzhou University, Jiangsu, 225009, China

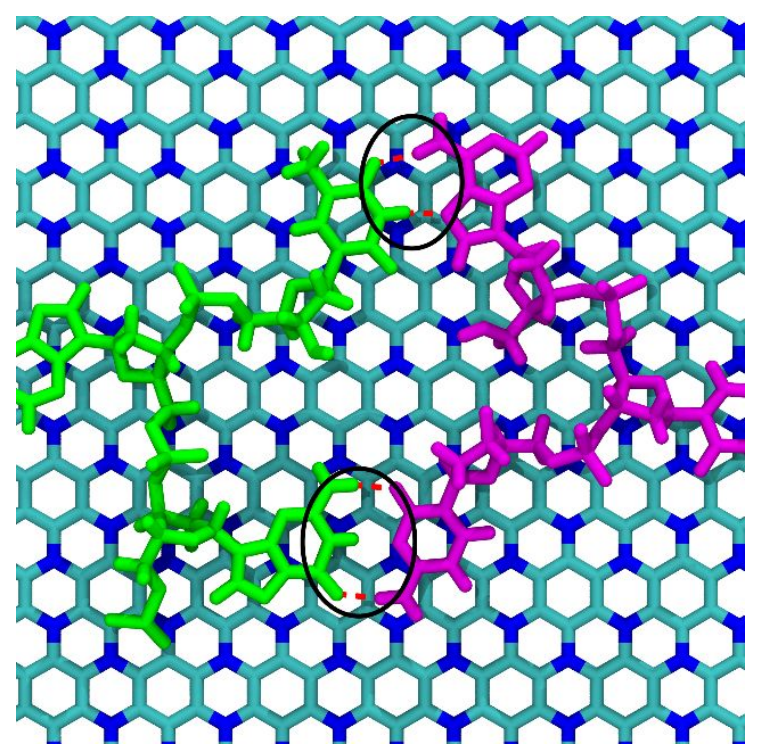

Figure S1. The hydrogen bonds partially remained on $\mathrm{C}_{3} \mathrm{~N}$ surface. Red dashed lines showed the hydrogen bonds, which were indicated by black circle. The conformation was obtained from the traj 3 of dsDNA $\perp \mathrm{C}_{3} \mathrm{~N}$. 


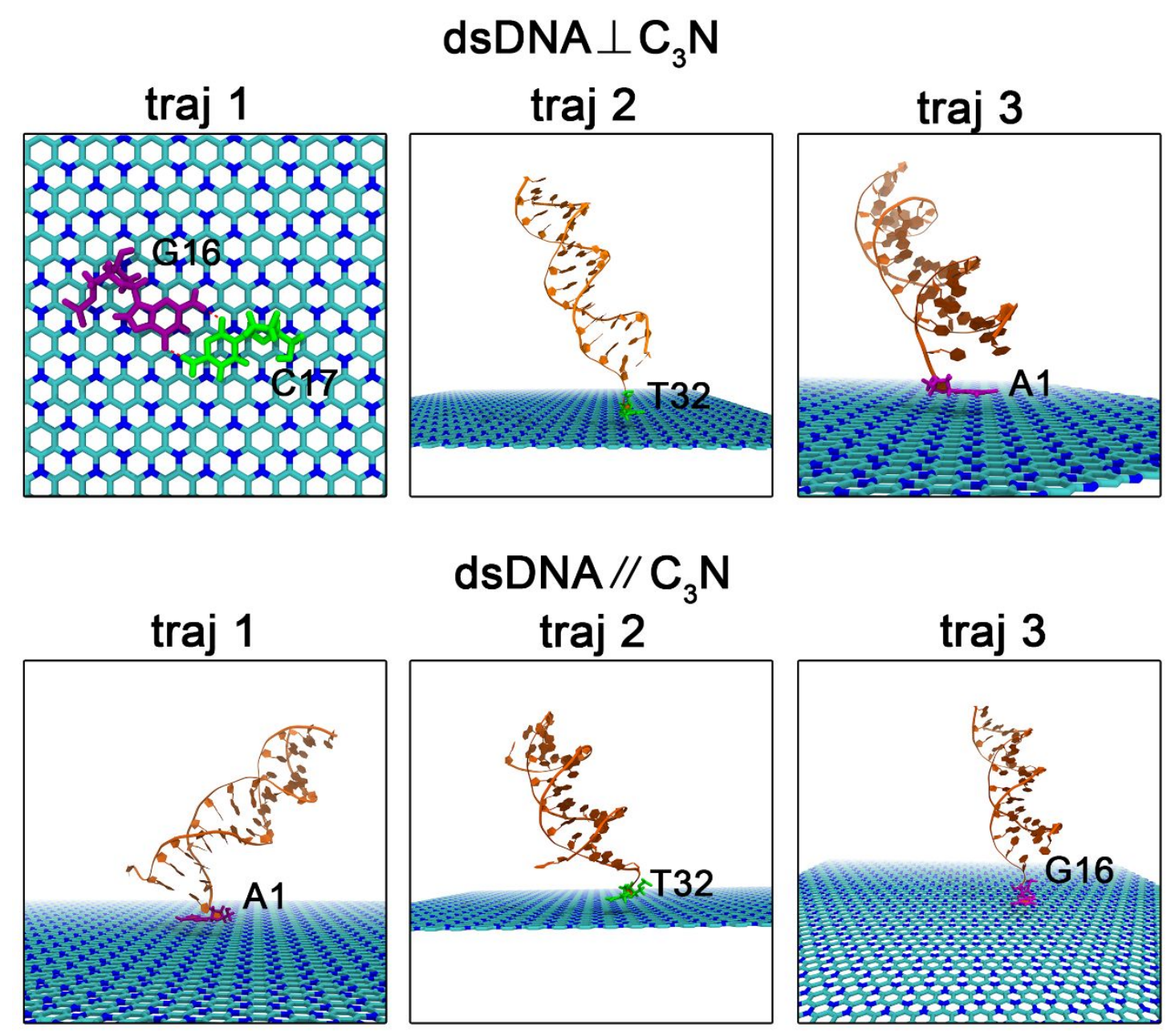

Figure S2. The intact interfacial base-pair in traj 1 of dsDNA $\perp \mathrm{C}_{3} \mathrm{~N}$. The red dashed lines indicated the hydrogen bonds. The initial connection of one nucleotide to $\mathrm{C} 3 \mathrm{~N}$ in traj 2 and 3 of dsDNA $\perp$ $\mathrm{C}_{3} \mathrm{~N}$ as well as in traj 1,2 and 3 of dsDNA// $\mathrm{C}_{3} \mathrm{~N}$. The key interfacial nucleotides were shown by purple and green sticks. 
a
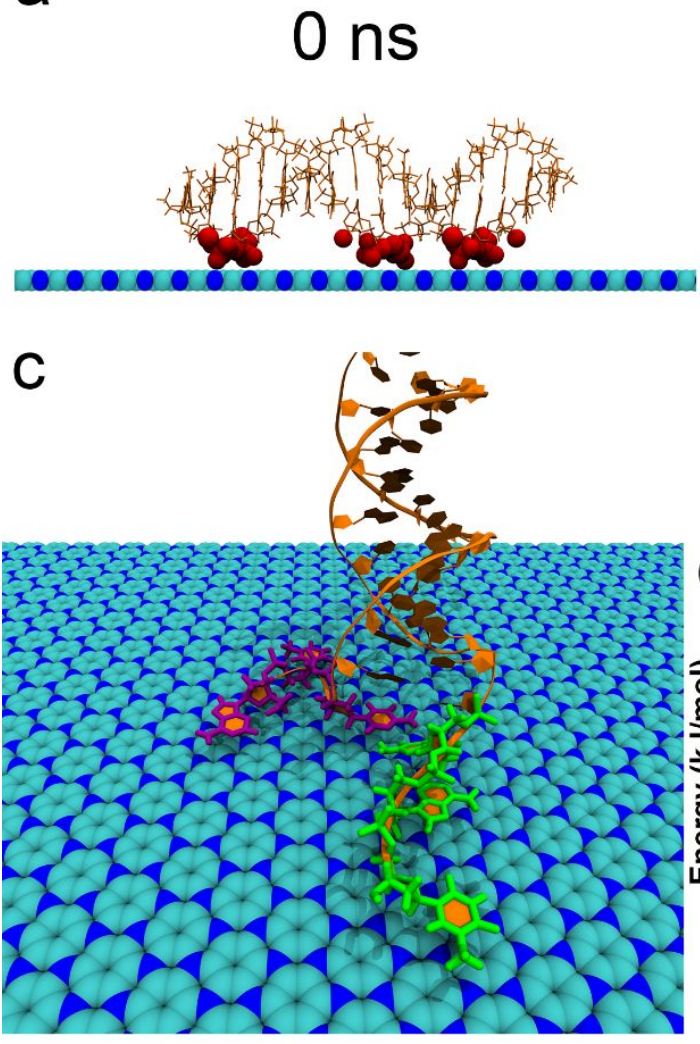

b

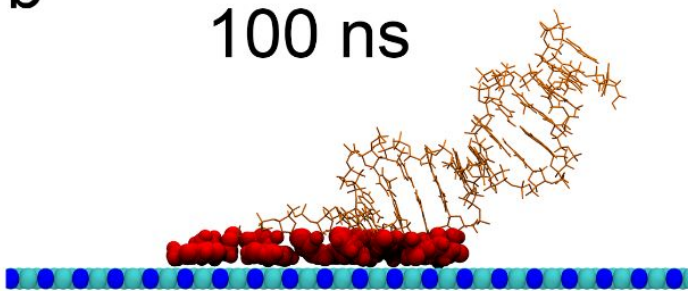

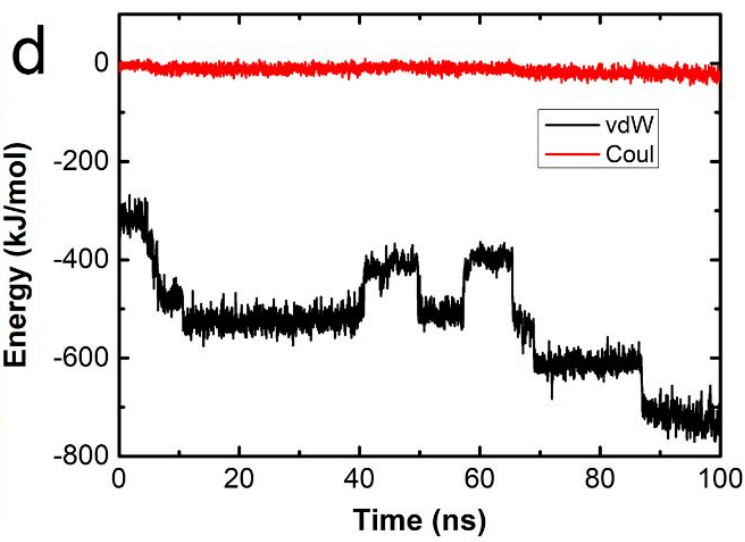

Figure $\mathrm{S} 3$. Simulation of dsDNA// $\mathrm{C}_{3} \mathrm{~N}$ with dsDNA backbone directly contacting to $\mathrm{C}_{3} \mathrm{~N}$. (a) Initial simulation setup. The red spheres indicated the contacted atoms of dsDNA to $\mathrm{C}_{3} \mathrm{~N}$ (with a cut-off of $0.6 \mathrm{~nm}$ ). (b) The binding conformation in $100 \mathrm{~ns}$. (c) The binding conformation of interfacial nucleotides on $\mathrm{C}_{3} \mathrm{~N}$. (d) Interaction energies between dsDNA and $\mathrm{C}_{3} \mathrm{~N}$.

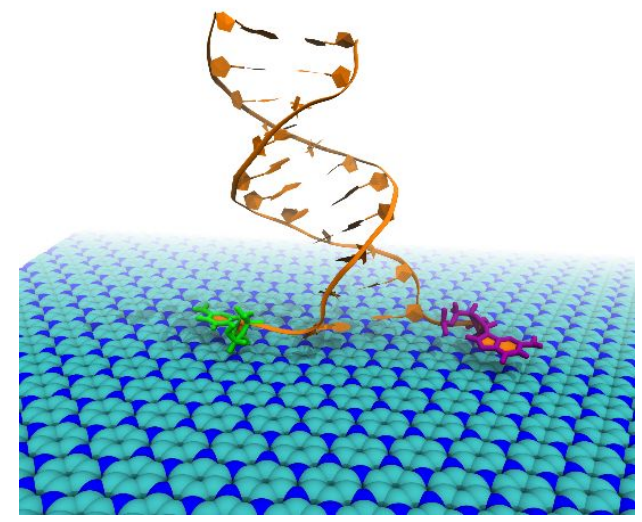

Figure S4. Final conformation of a real dsDNA binding to $\mathrm{C}_{3} \mathrm{~N}$ nanosheet. 

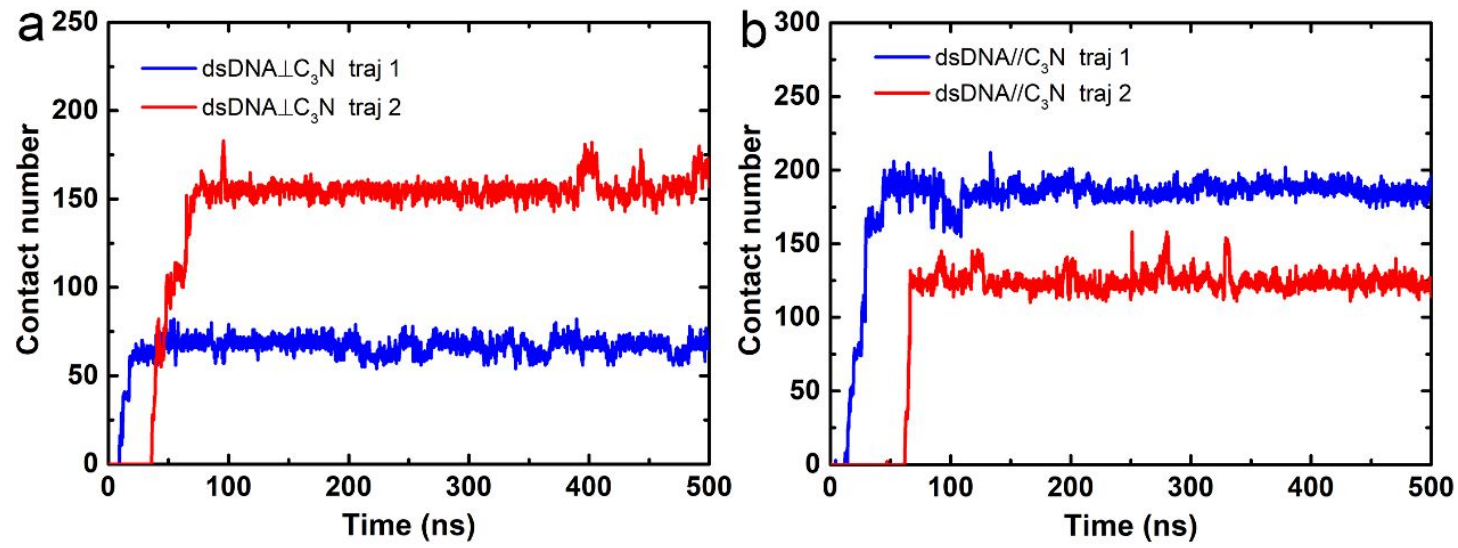

Figure S5. The contact number evolutions of dsDNA binding to $\mathrm{C}_{3} \mathrm{~N}$ in four trajectories.
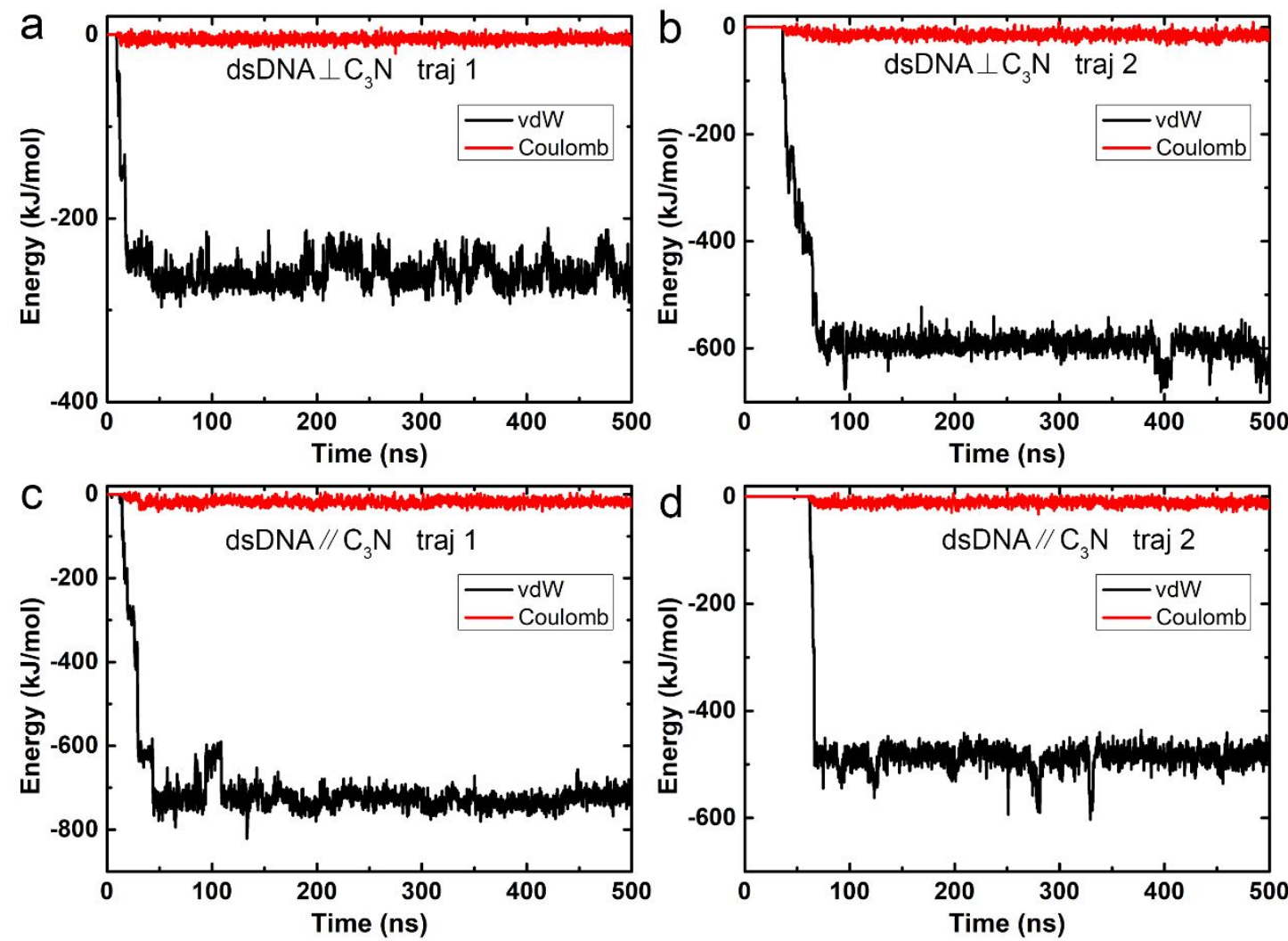

Figure S6. The interaction energies (including vdW and Coulomb contributions) of the dsDNA absorption process to the $\mathrm{C}_{3} \mathrm{~N}$ nanosheet in four trajectories. 

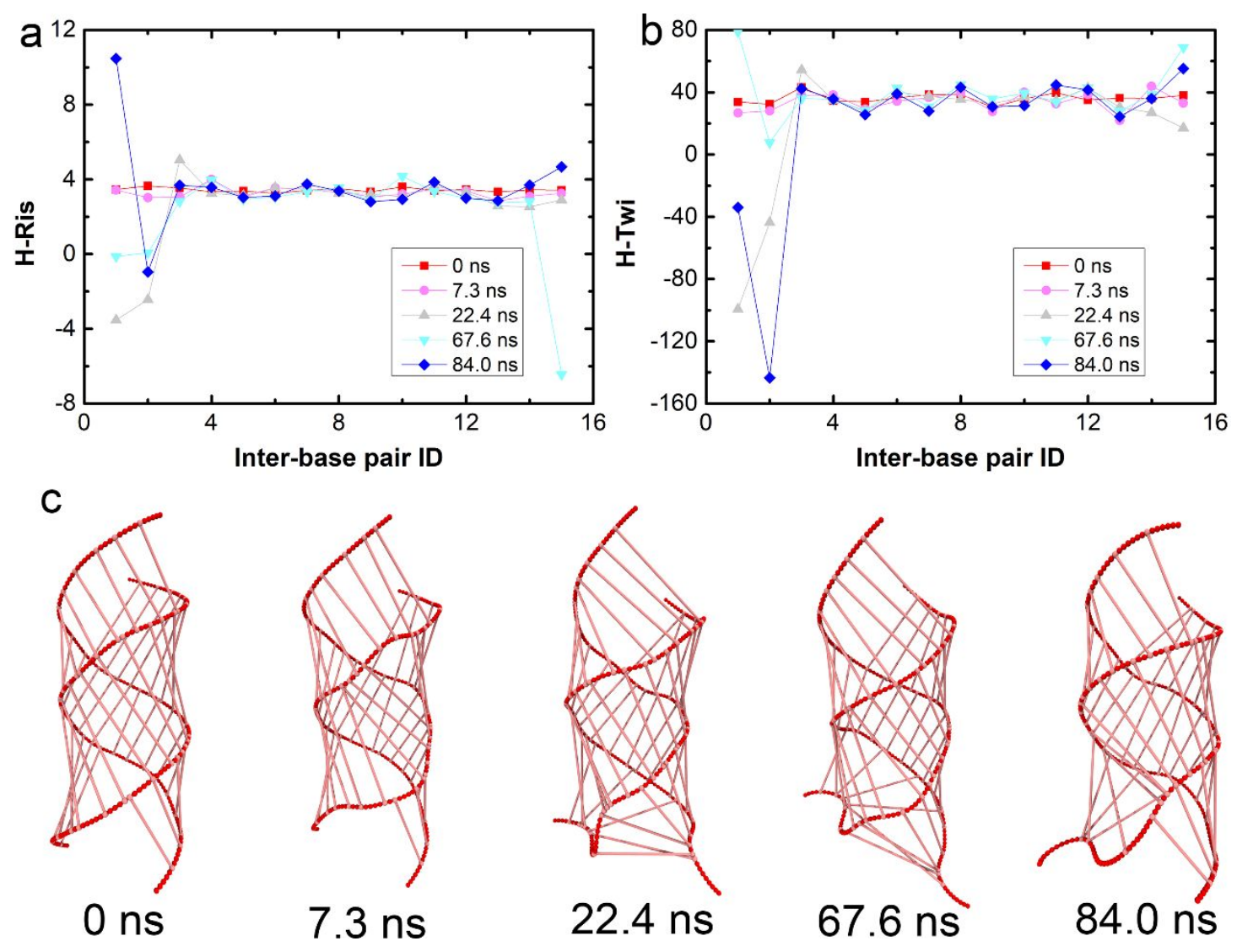

Figure S7. Structural changes of dsDNA corresponding to Fig. 4. (a) H-Ris corresponded to the translation of successive base pairs along the helical axis. (b) H-Twi corresponded to the rotation of successive base pairs around the helical axis. (c) The grooves of dsDNA. The pink sticks indicated the grooves of dsDNA whereas the red spheres represented the dsDNA backbone. 

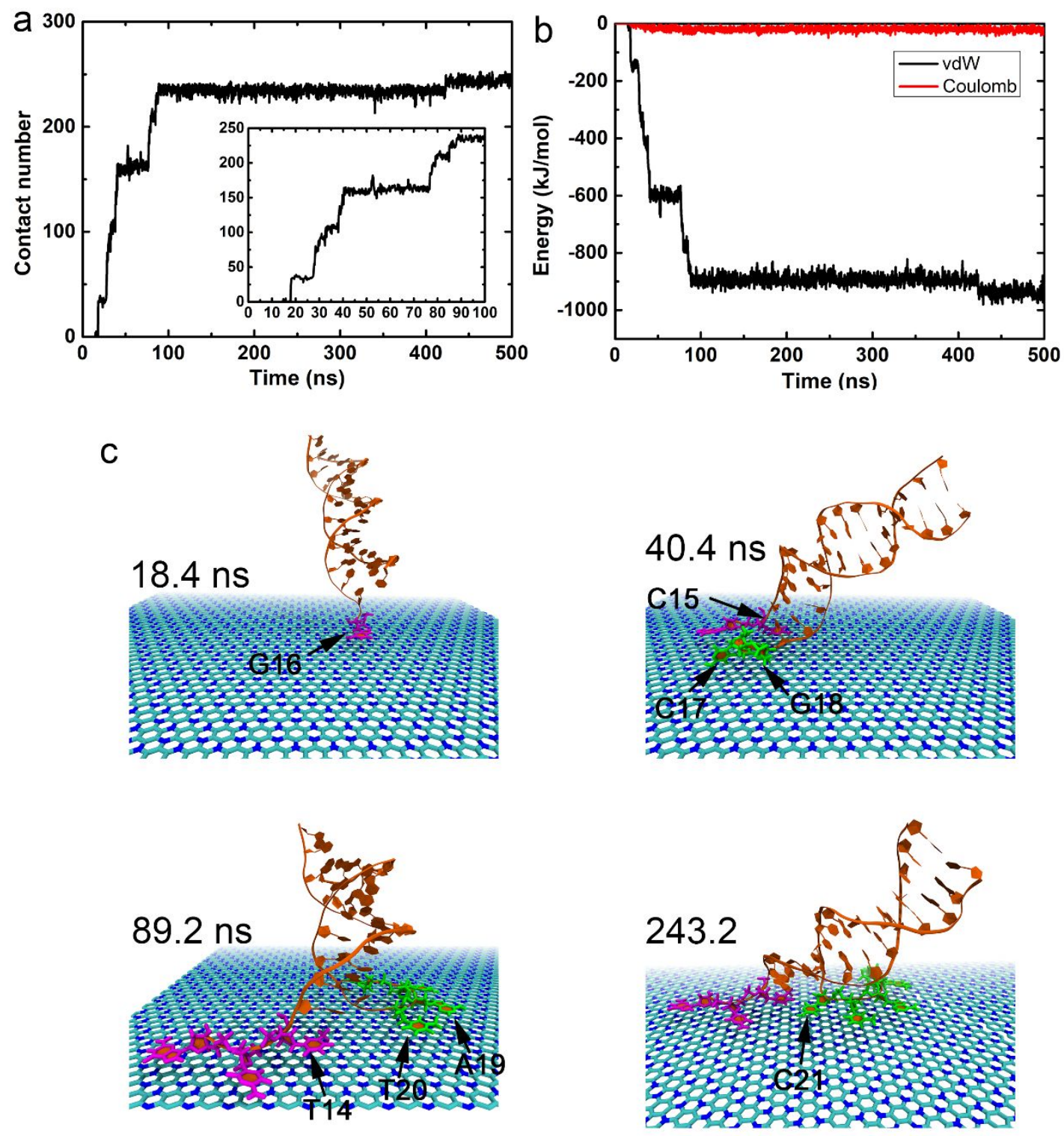

Figure S8. Kinetics of dsDNA unwinding on $\mathrm{C}_{3} \mathrm{~N}$ nanosheet by choosing a representative trajectory

(traj 3) of dsDNA// $\mathrm{C}_{3} \mathrm{~N}$ system. Time-evolutions of contact number (a) and interaction energies (including vdW and Coulomb contributions) (b) of the dsDNA absorption process to the $\mathrm{C}_{3} \mathrm{~N}$ nanosheet. (c) Key snapshots of the binding and unwinding process. Some important nucleotides were also displayed. 


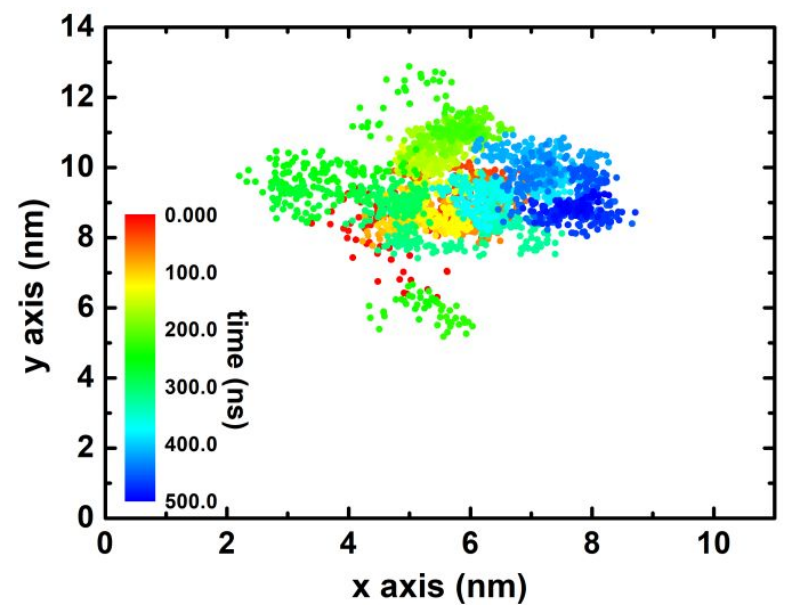

Figure S9. The center of mass (CoM) of dsDNA projected to $x-y$ plane $\left(\mathrm{C}_{3} \mathrm{~N}\right.$ surface). The data was collected from the traj 3 of dsDNA $\perp \mathrm{C}_{3} \mathrm{~N}$ system.

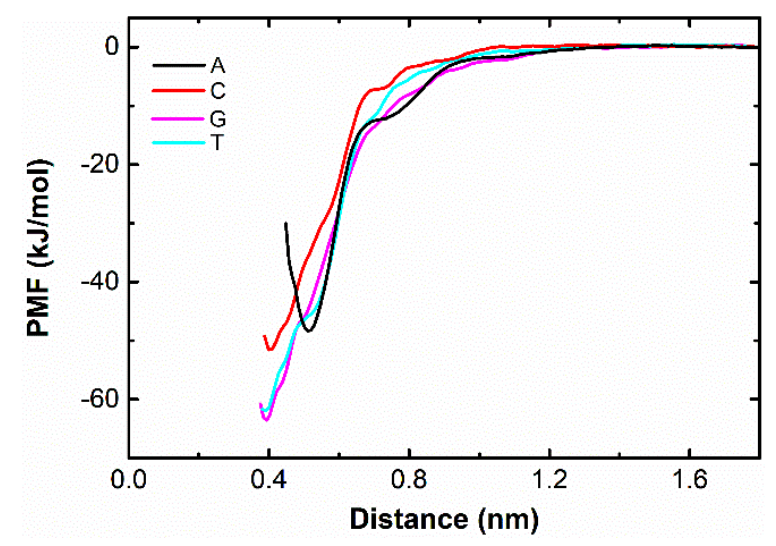

Figure S10. Potential of mean forces (PMFs) of four nucleotides binding to $\mathrm{C}_{3} \mathrm{~N}$ nanosheet. 